\title{
Early results from AIRS on the EOS
}

Hartmut H. Aumann, Thomas S. Pagano

Hartmut H. Aumann, Thomas S. Pagano, "Early results from AIRS on the EOS," Proc. SPIE 4881, Sensors, Systems, and Next-Generation Satellites VI, (8 April 2003); doi: 10.1117/12.462605 


\title{
Early Results from AIRS on the EOS
}

\author{
H.H. Aumann and Tom Pagano \\ California Institute of Technology , 4800 Oak Grove Drive, Pasadena CA 91109 \\ Jet Propulsion Laboratory \\ Pasadena, California
}

\begin{abstract}
The Atmospheric Infrared Sounder (AIRS) on the EOS Aqua spacecraft is an infrared spectrometer/radiometer which covers the $650-2700 \mathrm{~cm}^{-1}$ region of the spectrum with 2378 spectral channels. The EOS Aqua was launched on 4 May 2002 from Vandenburg AFB, California, into a $705 \mathrm{~km}$ high, sun synchronous orbit. First tests of the radiometric calibration using the analysis of (observed - calculated ) for data from a single, relatively cloud free $2500 \times 2500 \mathrm{~km}$ area of the subtropical Atlantic ocean confirm absolute radiometric accuracy of better than $0.5 \mathrm{~K}$. The spectral information in the data also suggests that the analyzed region contained more moisture than the NCEP analysis. Based on these results we expect that the assimilation of AIRS data into the forecast to result in major medium range weather forecast improvements and that the data set recorded by AIRS during its nominal seven year lifetime will be a major resource for climate studies.
\end{abstract}

Keywords: Hyperspectral, infrared, climate, radiometric calibration, weather-forecasting.

\section{INTRODUCTION}

The Atmospheric Infrared Temperature sounder (AIRS) was launched on 4 May 2002, as a key facility instrument on the EOS Aqua satellite. The AIRS objective is to provide sounding data for operational numerical weather forecasting and radiometrically calibrated spectra in support of NASA's climate and process research. AIRS is a grating array spectrometer which covers the spectral range from 650 to $2700 \mathrm{~cm}^{-1}$ (3.7 to 15.4 microns) with 2378 spectral channels and spectral resolution $\Delta \lambda / \lambda=1200$. In order to optimize the performance as a radiometer, the spectrometer optical elements are passively cooled to $150 \mathrm{~K}$, with active control at the $0.01 \mathrm{~K}$ level. Detector performance is optimized by cooling the detectors with a Sterling type refrigerator to 58K. Background information on AIRS are given by Aumann et al. (2003).

For the purpose of radiometric calibration the AIRS spectrometer is a simple cross-track scanning radiometer with 2378 spectral channels. Each scan line consists of 90 scene footprints (1.1 degree, corresponding to a $13.5 \mathrm{~km}$ diameter at nadir), a calibration using a full aperture wedge blackbody calibration source set to $308 \mathrm{~K}$ (the OBC), and four cold space views (SV) for every scan line. Each scan line repeats ever 2.667 seconds. During the scan line the spacecraft moved one footprint diameter forward in the orbit. A radiometrically calibrated map of the ground is thus obtained at the 2378 frequencies. The gain of each of the 2378 AIRS channels is independently determined and the noise of the detectors is uncorrelated. The radiometric correlation due to the common use of the $\mathrm{OBC}$ and the use of the same cold space views for the entire scan line is inherent to this design. By including a number of second order terms, such linearity correction, scan mirror temperature and scan angle dependent polarization effects, the quality of the absolute radiometric calibration can be improved to reach laboratory standards. An extensive pre-launch calibration effort using a NIST traceable blackbody demonstrated absolute radiometric calibration accuracy at the better than $0.2 \mathrm{~K}$ level for scene temperatures between $215 \mathrm{~K}$ and $315 \mathrm{~K}$ (Pagano et al. 2000). This excellent performance is made possible by the fact that the AIRS spectrometer is cooled to $150 \mathrm{~K}$, i.e. well below the coldest temperature expected to be measured in the Earth scene. The better than $0.2 \mathrm{~K}$ radiometric calibration is matched by low instrument noise, measured as Noise Equivalent Delta Temperature (NEDT), which is of the order or $0.2 \mathrm{~K}$ or less for most AIRS channels for each footprint. The AIRS radiometric calibration algorithm is described in the AIRS Calibration Theoretical Basis Document (ATBD) (Aumann et al. 2000). 
AIRS first light data were received on 14 June 2002. A full 24 hours of data from 20 July 2002 was made available to the AIRS science team for the initial evaluation of radiometric and spectral performance and evaluation of noise characteristics. In the following discussion we report on the use of these data for the verification of the AIRS radiometric calibration.

\section{APPROACH}

Due to uncertainties in the ground truth and cloud contamination effects, the verification of radiometric performance using Earth view data at the $0.2 \mathrm{~K}$ level achieved during pre-launch calibration is a major challenge. Various approaches are being evaluated by the AIRS science team. The one we have used for the early evaluation is limited to tropical and subtropical ocean at night and requires two steps: 1. Identify footprints which are free of clouds, without assuming the validity of the absolute calibration. 2 . Use the sea surface temperature from a model to establish radiometric performance in reasonably transparent areas of the spectrum by analyzing (observed - calculated). For the identification of footprints which are free of clouds over water we reject pixels where the land fraction, calculated for each pixel based on the known scan angle and spacecraft position, exceeded $1 \%$. We then use a spatial coherence test (SCT) which makes two assumptions: 1. We assume that any footprint where the brightness temperature measured in a good atmospheric window is lower that $280 \mathrm{~K}$, can be rejected. 2 . We assume that at night the tropical and subtropical ocean temperatures are uniform at the measurement noise level and on a $45 \mathrm{~km}$ scale, i.e. in the absence of clouds the brightness temperature measured by a good surface channel and its eight surrounding pixels, which cover a $45 \mathrm{~km}$ spot on the ground, should agree within the noise. Any low noise surface channel will do for this test. We selected the $2616 \mathrm{~cm}^{-1}$ super window channel. Since its NEDT $=0.1 \mathrm{~K}$ at $300 \mathrm{~K}, 90 \%$ of the measurements of adjacent footprints should agree within $0.25 \mathrm{~K}$ for a uniform scene. Other than the $280 \mathrm{~K}$ rejection threshold the SCT makes no assumptions about the validity of the absolute calibration, but the data analysis assumes that a reasonable fraction of the footprints is clear. Our unit of data is one granule, defined as six minutes of AIRS data. One granule covers an area of about $2500 \times 2500 \mathrm{~km}$ with 12150 footprints in 135 scan lines. We make sure that the "reasonably clear" assumption is satisfied by sampling each granule with the SCT and by selecting only those granule which are more than $50 \%$ ocean with at least 500 "SCT clear" pixels.
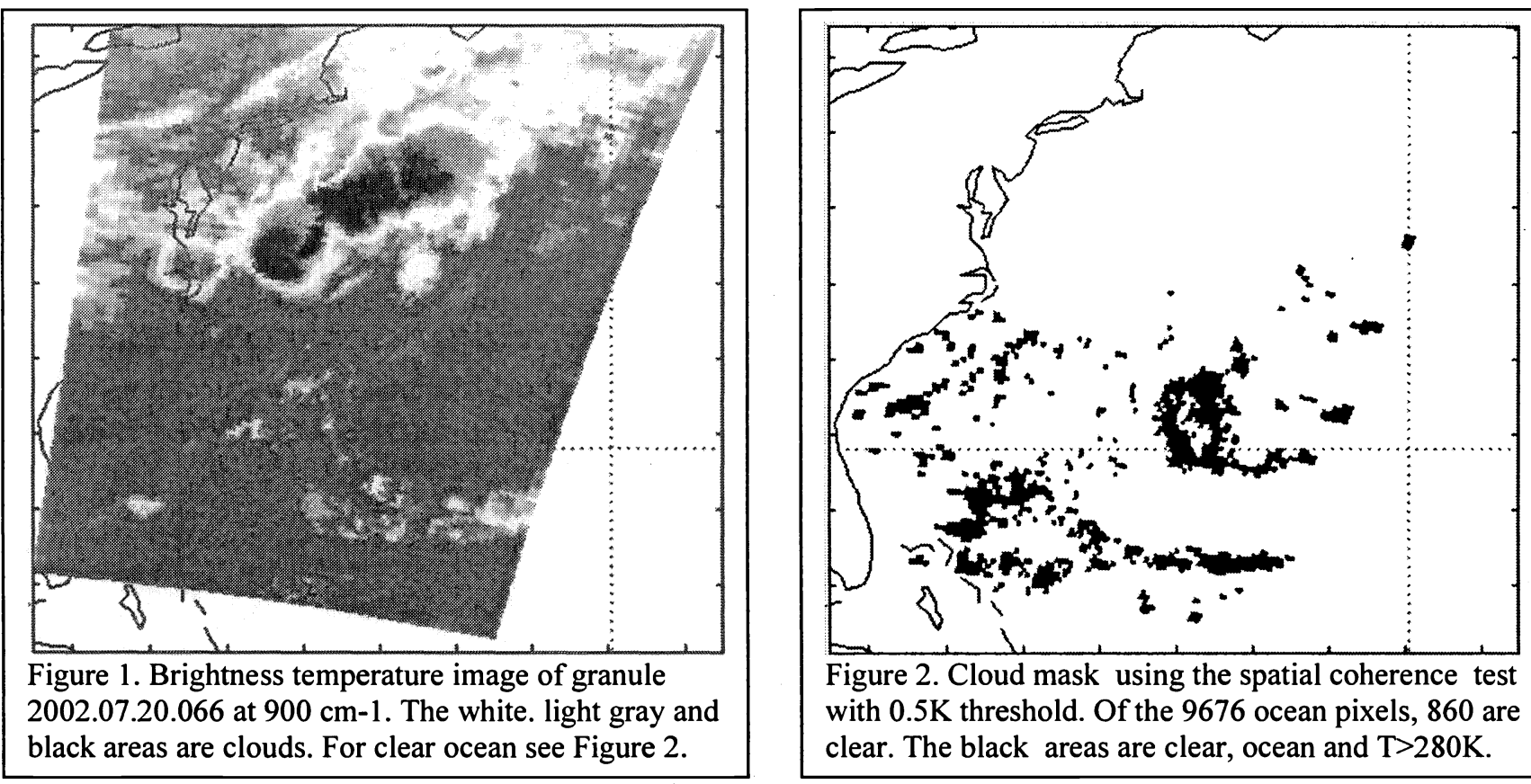

Figure 2. Cloud mask using the spatial coherence test with $0.5 \mathrm{~K}$ threshold. Of the 9676 ocean pixels, 860 are clear. The black areas are clear, ocean and $\mathrm{T}>280 \mathrm{~K}$. 


\section{RESULTS}

For the first evaluation of the radiometric performance of AIRS we selected the least cloudy subtropical ocean granule for 20 July 2002. Figure 1 . shows the brightness temperature at $900 \mathrm{~cm}^{-1}$ of granule 2002.07.20.066 with the coastline map overlaid. This granule covers a large part of the Atlantic Ocean north of Cuba and east of Florida. Figure 2. shows a map of the pixels identified by the SCT as ocean and clear using a spatial coherence test with the $0.5 \mathrm{~K}$ threshold.

Of the 12150 pixels in the granule, 9676 were identified as ocean with $\mathrm{T}>280 \mathrm{~K}$. Using SCT thresholds of $0.25 \mathrm{~K}, 0.5 \mathrm{~K}$ and $0.75 \mathrm{~K}$, which identify 164,860 and 1670 clear pixels at a median skin temperature of $299.63,299.51$, and 299.36 , respectively, yields an estimate of residual cloud contamination. Since extrapolating to zero suggests that $299.7 \mathrm{~K}$ is clear, $\mathrm{SCT}(0.25 \mathrm{~K})$ and $\mathrm{SCT}(0.5 \mathrm{~K})$ may have about $-0.1 \mathrm{~K}$ and $-0.2 \mathrm{~K}$ of cloud contamination. It is interesting to note that the yield of "cloud-free" pixels at the $0.2 \mathrm{~K}$ level, i.e. near the AIRS random noise, is very low for the clearest night ocean granule of 20 July 2002 . For SCT $(0.25 \mathrm{~K})$ and SCT $(0.5 \mathrm{~K})$ only $1.5 \%$ and $9 \%$, respectively, are reasonably clear. In the following we use $\mathrm{SCT}(0.5 \mathrm{~K})$ for the statistical evaluation, realizing that it potentially is cold biased by $0.2 \mathrm{~K}$.

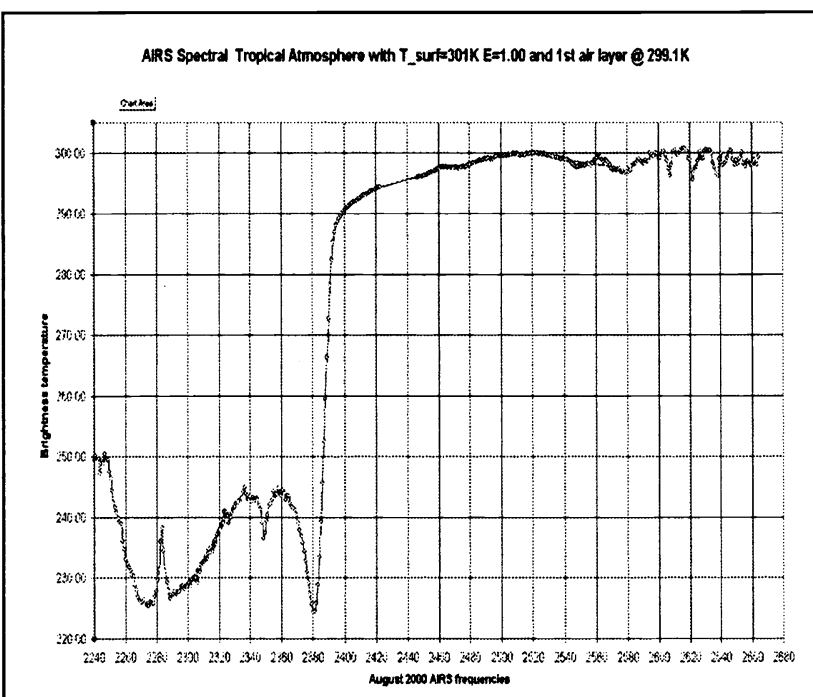

Figure 3. Calculated spectrum for tropical atmosphere.

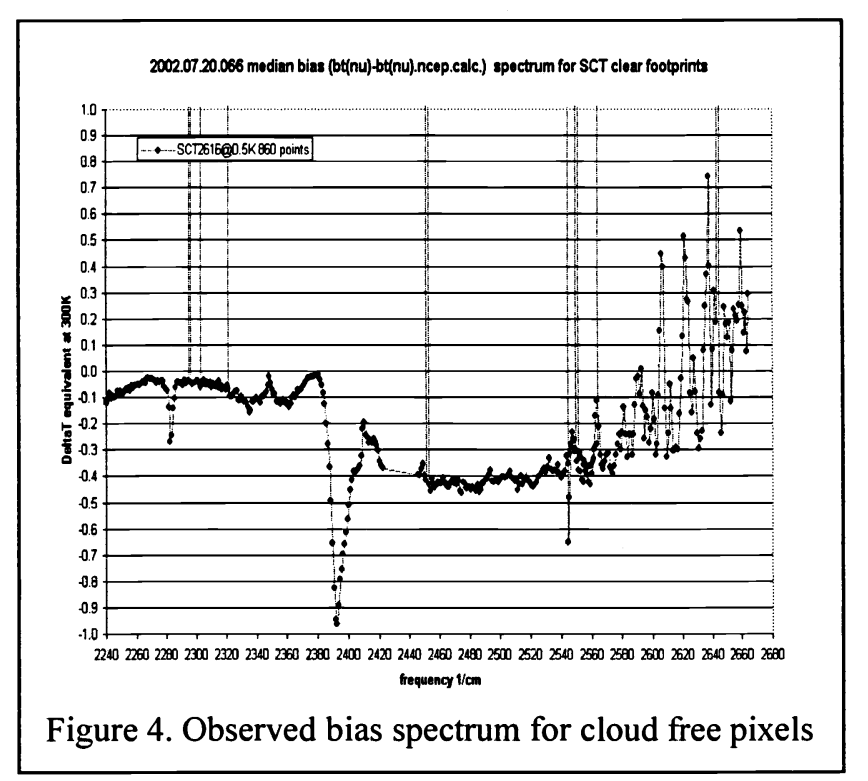

Bias spectra: The evaluation (observed - calculated) at night under cloud free conditions produces bias spectra. For those footprints identified as clear by the SCT we evaluate the statistics of $(b t(v)-\operatorname{sbt}(v)$.ncep), where bt( $v)$ is the brightness temperature measure by AIRS at frequency $v$, and $\operatorname{sbt}(v)$.ncep is the top-of-atmosphere (TOA) corrected brightness temperature at frequency $v$ calculated based on $T(p), q(p)$ and the SST in the NCEP model. The procedure for calculating the AIRS radiances is described by Fishbein (2003). It uses the radiative transfer algorithm (RTA) developed by Strow (2003) and the T(p), q(p) from the NCEP analysis grid, interpolated to the AIRS footprint positions. In the following we focus on bias spectra from the more transparent portions of the atmosphere in the 2500 and $900 \mathrm{~cm}^{-1}$ regions of the spectrum.

Figure 3. shows a calculated spectrum of $\operatorname{sbt}(v)$.ncep between 2240 and $2690 \mathrm{~cm}^{-1}$. The bias spectrum of (bt(nu)sbt(nu).ncep) for pixels identified as "clear" is shown in Figure 4. Dotted lines going vertically off scale are bad channels. The calculations are carried out in radiance units, and the difference radiance is converted to the equivalent temperature difference for a scene at a temperature of $300 \mathrm{~K}$. All data fit between \pm 1 , with the majority between $\pm 0.5 \mathrm{~K}$. What appear to be random scatter are, on closer inspection, highly correlated deviations which correlate with water and $\mathrm{CO}_{2}$ spectral features.

The most obvious spectral feature in Figure 4. near $2390 \mathrm{~cm}^{-1}$ is not related to calibration, but is due to the use of incorrect R-branch calculations in the pre-launch RTA. This is discussed by Strow et al. (2002). In the 2240 through 2380 $\mathrm{cm}^{-1}$ region, where the temperature ranges from $220 \mathrm{~K}$ to $250 \mathrm{~K}$ because the channels never see the surface, the bias is less than $0.1 \mathrm{~K}$. This can only be interpreted as meaning that the calibration in this region is excellent, the transmission of $\mathrm{CO}_{2}$ is well understood, and the temperature profile in the NCEP model is very accurate. In the highly transmitting region between 2440 and $2680 \mathrm{~cm}-1$ there is a bias between -0.4 and $-0.3 \mathrm{~K}$, except in the water lines, i.e. the observed is 
colder than expected (calculated). Since the NCEP SST is tied to the temperature of the buoys (at about 6 meter depth),

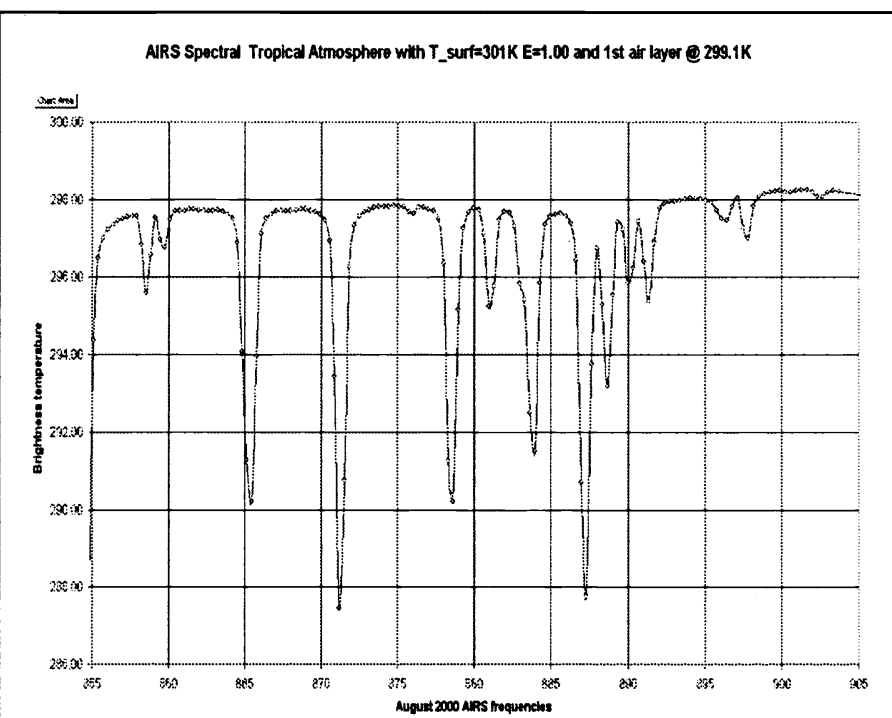

Figure 5. Calculated brightness temperature spectrum between 855 and $905 \mathrm{~cm}-1$.

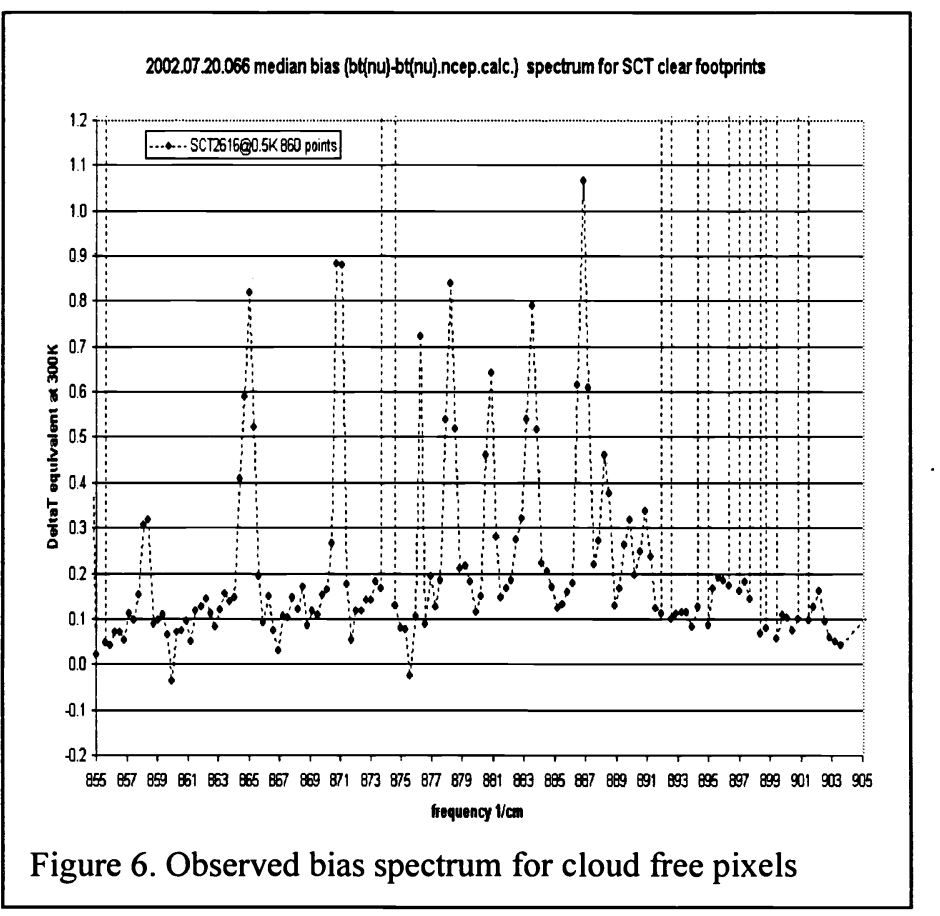

while AIRS measures the skin temperature in the upper few microns of the water surface, a small cold bias would be expected. The mean difference between ATSR $\mathrm{SST}_{\text {skin }}$ and TOA corrected SST $\mathrm{Suoy}_{\text {at night and wind }}$ speed $>6 \mathrm{~m} / \mathrm{sec}$ is $-0.17+/-0.46 \mathrm{~K}$ (Donlon et al. 2002), with values as low as $-0.6 \mathrm{~K}$ at lower wind speeds. The determination of absolute calibration for non-global data using the SST is thus limited by the uncertainty in the (skin - buoy) correction, in addition the regional $0.5 \mathrm{~K}$ SST model accuracy. Both uncertainties are due to differences in regional conditions of sea state and evaporation from the surface, which average out in global calculations.

Figure 5. shows a calculated spectrum of $\operatorname{sbt}(v)$.ncep between 855 and $905 \mathrm{~cm}^{-1}$. The bias spectrum of (bt $(v)$-sbt $(v)$.ncep) for pixels identified as "clear" is shown in Figure 6. For the moment we will ignore the interesting bias spectrum which mimics the structure of the water lines shown in Figure 5 and focus on the bias between the lines. The bias in this region is typically $+0.1 \mathrm{~K}$. This is inconsistent with what we saw in Figure 4. Since the change in water emissivity between 2500 and $900 \mathrm{~cm}-1$ is already included in the calculated radiances, we would have expected the bias in the two spectral regions to be the same. Instead we find a bias of $0.4 \mathrm{~K}$ between the 2616 and $900 \mathrm{~cm}-1$ window channels.

Given the excellent agreement between observed and calculated in the 2240 through $2380 \mathrm{~cm}^{-1}$ region, this difference is significant. If we postulate that the AIRS calibration is correct at other frequencies as well, we have three potential explanations for the $0.4 \mathrm{~K}$ bias: 1 . Residual cloud contamination. 2. The TOA correction at $2616 \mathrm{~cm}-1$ is incorrect, and/or 3. the TOA correction at $900 \mathrm{~cm}-1$ is incorrect. There may well be residual cloud contamination at the $0.2 \mathrm{~K}$ level due to the SCT $(0.5 \mathrm{~K})$, but it would make both spectral regions look colder. Regarding explanation 2) we note that the TOA correction at $2616 \mathrm{~cm}-1$ is very small. It can be expressed as bt.toa $=$ sst.ncep $-0.8-0.3 / \cos$ (theta), where theta is the local slant path angle and the $0.8 \mathrm{~K}$ adjusts for the emissivity of the ocean with $0.2 \mathrm{~K}$ uncertainty (Masuda et al. 1988 and VanDelst and Wu 2000). At $900 \mathrm{~cm}-1$ the emissivity of the ocean is 0.99 under almost any conditions. Half the observed difference between 900 and $2616 \mathrm{~cm}^{-1}$ could be due to emissivity uncertainty at $2616 \mathrm{~cm}^{-1}$. Suggestion 3 . is the most likely. The correction for water vapor absorption in the most transparent channel in this region, $900 \mathrm{~cm}^{-1}$, is about $3 \mathrm{~K}$ at nadir for tropical ocean climatology. A $10 \%$ error in the amount of water vapor in the model for the clear footprints would thus introduce a $0.3 \mathrm{~K}$ shift in the expected temperature, which would account for almost the entire observed difference. There is direct evidence for possibility 3 ) in the form of the large bias in the water lines. At the center of the lines the observed temperature is considerably warmer than the 
calculated temperature, suggesting that either there is about $10 \%$ too much water in the average NCEP analysis for the clear pixels in this particular data granule, or that the transmission due to water vapor is overcorrected by $0.4 \mathrm{~K}$.

\section{CONCLUSIONS}

Based on the analysis of a single, relatively cloud free $2500 \times 2500 \mathrm{~km}$ granule of AIRS data taken on 20 July 2002 , we conclude that the absolute radiometry in reasonably transparent parts of the AIRS spectrum is within $0.5 \mathrm{~K}$ of the NCEP analysis, but could be considerably more accurate. Validation of the calibration approaching the $0.2 \mathrm{~K}$ accuracy claimed based on pre-launch calibration is complicated by the effects of residual cloud contamination, uncertainty in the surface temperature, surface emissivity and TOA correction. Residual cloud contamination can be reduced to the $0.1 \mathrm{~K}$ level using the SCT( $0.25 \mathrm{~K})$, but the yield of "cloud-free" pixels is very low. Assuming that the SST model and water vapor column model data are globally unbiased, the buoy temperature and the water vapor column uncertainty in the TOA correction should average out on a global basis. On a global basis the absolute calibration is thus limited to the accuracy of the assumed (bulk-skin) bias and a frequency dependent bias in the emissivity.

The fact that the absolute calibration of AIRS is within $0.5 \mathrm{~K}$ of surface truth using essentially first light data is a tribute to the design of AIRS, the radiometric precision achievable with a cooled grating array radiometer, the power of radiometry with high spectral resolution and broad spectral coverage. Observations from this single granule of data only confirm that the absolute calibration of AIRS is good to within $0.5 \mathrm{~K}$, the calibration may well be considerably better. With the analysis of globally well distributed data we expect to be able to validate the AIRS absolute radiometric calibration accuracy on-orbit to within the same $0.2 \mathrm{~K}$ level demonstrated during pre-launch testing and to monitor it throughout the expected seven year life of the EOS Aqua mission. The availability of routine high spectral resolution observations with laboratory quality radiometric precision from Earth orbit will make a major contribution to climate research and weather forecasting.

\section{ACKNOWLEDGEMENTS}

This work was carried out at the Jet Propulsion Laboratory, California Institute of Technology under contract from the National Aeronautics and Space Administration. The AIRS instrument was developed for the Earth Observing System by Lockheed Martin (now BAE Systems) under contract with the Jet Propulsion Laboratory. Dr. Evan Fishbein, JPL, provided helpful insight into the calculation of the expected cloud-free radiances using the NCEP model calculations and the uncertainty of the sea surface emissivity.

\section{REFERENCES}

1. Aumann, H. H., M. T. Chahine, C. Gautier, M. Goldberg, E. Kalnay, L.C. McMillin, H. Revercomb, P.W. Rosenkranz, W.L. Smith, D. Staelin, L. Strow and J. Susskind,"AIRS/AMSU/HSB on the Aqua Mission: Design, Science Objectives, Data Products and Processing Systems", accepted for TGARSS Special Issue on EOS Aqua. March 2003. Also see http://airsteam.jpl.nasa.gov for up-to-date technical information on AIRS.

2. Pagano T. S., Hartmut H. Aumann and Larrabee Strow, "Pre-launch Performance Characteristics of the Atmospheric Infrared Sounder (AIRS)", SPIE 4169-41, (2000).

3. Aumann, Hartmut .H., David T. Gregorich, Steven L. Gaiser, Denise F. Hagan, T. S. Pagano, Larrabee Strow and Dean Ting, "AIRS Level 1b Algorithm Theoretical Basis Document (ATBD) Part 1 (IR)", 10 November 2000.

4. Fishbein, E., D. Gregorich, M. Gunson, M. Hofstadter, S.Y. Lee, P. Rosenkranz and L. Strow, "The Atmospheric Infrared Sounder (AIRS) Simulation System", accepted for TGARSS Special Issue on EOS Aqua. March 2003.

5. Strow, Larrabee, Scott Hannon, S. DeSouza Machado, H. Motteler, and David Tobin, "An Overview of the AIRS Radiative Transfer Model”, accepted for TGARSS Special Issue on EOS Aqua. March 2003.

6. Strow , Larrabee and S. DeSouza Machado, "Validation of the AIRS Radiative Transfer Algorithm using ECMWF Model Data", Proceedings of the SPIE EUROPTO September 2002, Crete, Greece. 
7. Donlon, C. J., P.J. Minnett, C. Gentemann, T.J. Nightingale, I.J. Barton, B. Ward, and J. Murray, "Towards Improved Validation of Satellite Sea Surface Skin Temperature Measurements for Climate Research", Journal of Climate, 15, 353369 (2002).

8. Masuda, K., T. Takashima, and Y. Takayama, "Emissivity of Pure and Sea Waters for the Model Sea Surface in the Infrared Window Region", Remote Sensing of the Environment, 24, 313-329 (1988).

9. VanDelst, P. and X. Wu, "A high resolution infrared sea surface emissivity database for satellite applications", Proc. of $11^{\text {th }}$ Intl. ATOVS Study Conference, 407, (2000). 